\title{
SIMULTANEOUS MULTIFRACTAL ANALYSIS OF THE BRANCHING AND VISIBILITY MEASURE ON A GALTON-WATSON TREE
}

\author{
ADAM L. KINNISON* AND \\ PETER MÖRTERS, ${ }^{* * *}$ University of Bath
}

\begin{abstract}
On the boundary of a Galton-Watson tree we can define the visibility measure by splitting mass equally between the children of each vertex, and the branching measure by splitting unit mass equally between all vertices in the $n$th generation and then letting $n$ go to infinity. The multifractal structure of each of these measures is well studied. In this paper we address the question of a joint multifractal spectrum, i.e. we ask for the Hausdorff dimension of the boundary points which simultaneously have an unusual local dimension for both these measures. The resulting two-parameter spectrum exhibits a number of surprising new features, among them the emergence of a swallowtail-shaped spectrum for the visibility measure in the presence of a nontrivial condition on the branching measure.
\end{abstract}

Keywords: Multifractal spectrum; two-parameter spectrum; mixed spectrum; twodimensional multifractal analysis; random tree; self-similar fractal; branching process; percolation

2000 Mathematics Subject Classification: Primary 60J80; 28A80

\section{Motivation}

Multifractal analysis provides a way of encapsulating complex information about the fractal nature of an object in a single curve, the multifractal spectrum. In this paper we show how a multifractal analysis can also offer deep insight into the relationship between two fractal objects in the form of a two-parameter multifractal spectrum. In particular, we shall see that when one of the analysed measures fails to obey the 'multifractal formalism', such an analysis can lead to the discovery of new phenomena which are deeply rooted in the geometry of these measures.

Our test case is the boundary of a Galton-Watson tree with nonzero offspring at every vertex. This set is, on the one hand, a familiar and well-studied object in probability and, on the other hand, it represents the symbolic dynamics of a class of self-similar random fractals and as such it is representative of the behaviour of a wider range of fractal objects with statistical self-similarity. Two natural measures can be defined on the boundary of a Galton-Watson tree, the visibility measure and the branching measure. Both have been studied separately from a multifractal point of view.

The visibility measure is easily defined, by starting at the root of the tree with a unit mass and, recursively, at each vertex splitting it equally among the children. If the offspring distribution of the Galton-Watson tree is nondegenerate and satisfies some mild moment conditions, the

Received 5 May 2009; revision received 30 December 2009.

* Postal address: Department of Mathematical Sciences, University of Bath, Bath BA2 7AY, UK.

** Email address: maspm@bath.ac.uk 
visibility measure is multifractal and the multifractal formalism (see, e.g. [11]) applies; see [16] for details. The branching measure represents the uniform measure on the boundary. It can be defined by taking the uniform distribution on the vertices in the $n$th generation and taking a limit as $n \rightarrow \infty$. This measure is not multifractal if the offspring variable has zero probability of taking the value 1 , and it is only multifractal in a weaker sense otherwise. Only unusually large values of the upper local dimension are possible, and can be represented in a spectrum of hyperbolic shape; see [28] and [37]. In this situation the multifractal formalism does not apply as it always leads to a concave spectrum.

Liu and Rouault [20] proved that the visibility and the branching measure are mutually singular as soon as the offspring random variable is nondegenerate. The aim of our analysis is to further clarify the relationship of the two measures and express the results in terms of a twoparameter multifractal spectrum, which gives the Hausdorff dimension of the set of points in the boundary, for which the local dimensions of the two measures are given by the two parameters. This 'simultaneous' multifractal analysis of the branching and visibility measures will unlock some surprising new features of the two measures and the way they interact. Our proofs involve a combination of the multifractal formalism and percolation techniques to establish the tricky lower bounds for the Hausdorff dimension.

\section{Background and main results}

\subsection{Background on multifractal analysis}

Multifractal spectra appeared first in the physics literature in papers by Mandelbrot [24], Benzi et al. [5], Frisch and Parisi [13], and Halsey et al. [14], who studied multifractal models that occur naturally in the world, in particular in the area of turbulence. For more examples of the use of multifractal spectra in these contexts, see the introduction to [18]. They have appeared in the mathematics literature with increasing regularity over the last 20 years. Examples appear in the analysis literature in the work of Rand [36], Brown et al. [8], Cawley and Mauldin [9], and Olsen [29] in the early 1990s, and more recently in the probability literature in the work of Arbeiter and Patzschke [2] in the case of random self-similar fractals, Perkins and Taylor [35] in the case of super Brownian motion, Mannersalo et al. [25] and Anh et al. [1] for products of stochastic processes, Berestycki [6] in the case of fragmentation processes, and Klenke and Mörters [17] in the case of intersection local time of Brownian motion. For some further examples of how multifractal spectra can improve our understanding about certain stochastic processes, see [26].

Recently, simultaneous, or 'mixed', multifractal spectra for several measures were discussed in the context of dynamical systems by Barreira et al. [3], [4] and, independently, in the context of geometric measure theory by Olsen [31]. Olsen introduced a general framework [32] and Olsen and Winter in [30] and [33] gave a complete analysis of deterministic self-similar measures satisfying the open set condition. These papers sought to generalize the 'multifractal formalism', established in the physics literature and verified in various mathematical setups, to a simultaneous multifractal analysis. The authors did not investigate the situation when the 'multifractal formalism' is violated by one of the involved measures. This situation however occurs frequently for random measures and in the context of measures defined in terms of stochastic processes, and is therefore of particular interest to us in the present paper.

We define multifractal spectra associated with the local dimensions (or Hölder exponents) of a fractal measure. To this end, let $\mu$ be a locally finite measure on a metric space $X$. We 
define the local dimension of the measure $\mu$ at a point $x \in X$ as

$$
\operatorname{dim}_{\mu}(x):=\lim _{r \downarrow 0} \frac{\log \mu(B(x, r))}{\log r},
$$

whenever the limit exists, where $B(x, r)$ is the closed ball of radius $r$ centred at the point $x \in X$. We also define the upper and lower local dimensions of $\mu$ at $x$ to be

$$
\overline{\operatorname{dim}}_{\mu}(x):=\limsup _{r \downarrow 0} \frac{\log \mu(B(x, r))}{\log r} \text { and } \quad \underline{\operatorname{dim}}_{\mu}(x):=\liminf _{r \downarrow 0} \frac{\log \mu(B(x, r))}{\log r},
$$

respectively. The local dimension indicates the rate at which the $\mu$-measure of the balls, centred at the point $x$, decay as the radii of the balls shrink to 0 . The rate of decay of the measure of the balls goes like the radius to the power of the local dimension. It follows that the larger the local dimension, the faster the measure of the balls decays. The local dimension may vary considerably between different points in the metric space. A measure $\mu$ is called monofractal if there exists a constant $\eta$ such that $\operatorname{dim}_{\mu}(x)=\eta$ for all $x \in \operatorname{supp} \mu$. Otherwise, the measure is often called multifractal.

If the measure $\mu$ is multifractal then its multifractal spectrum is the function

$$
f(\theta)=\operatorname{dim}\left\{x \in X: \operatorname{dim}_{\mu}(x)=\theta\right\},
$$

where dim normally denotes the Hausdorff dimension and $\theta$ ranges over all possible values of the local dimension. We are equally interested in the variants of this function arising when replacing the local dimension by upper or lower local dimensions, or when the equality inside the set is replaced by an appropriate inequality.

\subsection{Background on Galton-Watson trees}

Galton-Watson trees are characterised by a single parameter, the law of the offspring random variable $N$. Throughout this paper, we assume that $N$ is nondegenerate and takes values in the natural numbers, in particular it does not take the value 0 . We allow $N$ to be unbounded, but always assume that

$$
\gamma(\beta):=-\log \mathrm{E}\left[N^{\beta+1}\right]>-\infty \text { for all } \beta \in \mathbb{R} .
$$

We abbreviate $\mathfrak{m}:=\mathrm{E}[N]>1$ and $\mathfrak{a}:=\log \mathrm{E}[N]>0$.

The Galton-Watson tree $T$ is defined recursively. Start with the root $\rho$ which constitutes the 0 th generation. Given the tree up to the $n$th generation, sample an independent family $(N(v):|v|=n)$ of offspring variables indexed by the vertices in the $n$th generation of the tree, and attach precisely $N(v)$ children to vertex $v$. The union of these vertices then represent the $(n+1)$ th generation, and we define an infinite tree by proceeding ad infinitum.

Given a vertex $v$ in the $n$th generation, there exists a unique path $\rho=v_{0}, v_{1}, \ldots, v_{n}=v$ from the root to this vertex, such that $v_{i}$ is a child of $v_{i-1}$ for all $1 \leq i \leq n$. We sometimes identify $v$ with its ancestral line $\left(v_{0}, \ldots, v_{n}\right)$. A ray is an infinite sequence of vertices $v_{0}, v_{1}, \ldots$ such that $v_{0}=\rho$ and $\left(v_{0}, \ldots, v_{n}\right)$ is the ancestral line of $v_{n}$ for each $n \in \mathbb{N}$. By $\partial T$ we denote the boundary of the tree, consisting of all rays. We equip the boundary of the tree with the metric $d$ given by $d(u, v)=\mathrm{e}^{-n}$, where $n$ is the generation of the last common vertex of $u, v \in \partial T$. For $\xi=\left(v_{0}, v_{1}, \ldots\right) \in \partial T$, let $B\left(\xi, \mathrm{e}^{-n}\right)=B\left(v_{n}\right)$ be the closed ball with centre $\xi$ and radius $\mathrm{e}^{-n}$, which coincides with set of all rays containing the vertex $v_{n}$. 
For every vertex $v \in T$, we let $T(v)$ be the subtree consisting of all successors of $v$, and note that, conditional on $v \in T$, the tree $T(v)$ is again a Galton-Watson tree with the same offspring distribution. We denote by $Z_{n}(v)$ the number of vertices in the $n$th generation of (and relative to) $T(v)$, and abbreviate $Z_{n}:=Z_{n}(\rho)$.

The visibility measure $v$ on $\partial T$ is the probability measure defined by

$$
v(B(v))=\prod_{j=0}^{n-1} N\left(v_{j}\right)^{-1},
$$

where $\left(v_{0}, \ldots, v_{n}\right)$ is the ancestral line of the vertex $v$. Alternatively, $v$ is the law of the ray obtained as the path of a random walk starting at the root and moving at every step to a randomly chosen child of the current vertex. In [16] it was shown that, under our assumptions on the Galton-Watson tree, for every

$$
\sup _{\beta<0} \frac{-\gamma(\beta)}{\beta}<\eta<\inf _{\beta>0} \frac{-\gamma(\beta)}{\beta},
$$

we have, almost surely,

$$
\operatorname{dim}\left\{\xi \in \partial T: \operatorname{dim}_{\nu}(\xi)=\eta\right\}=\inf _{\beta}\{-\eta \beta-\gamma(\beta)\} .
$$

This spectrum is concave with a unique maximiser at

$$
\eta_{\text {typ }}:=\frac{\mathrm{E}[N \log N]}{\mathrm{E}[N]} .
$$

The proof of this result also shows that, for $\eta \leq \eta_{\text {typ }}$, the same spectrum holds when $\operatorname{dim}_{\nu}(\xi)=\eta$ is replaced by $\overline{\operatorname{dim}}_{v}(\xi) \leq \eta$, while, for $\eta \geq \eta_{\text {typ }}$, we may replace it by $\underline{\operatorname{dim}}_{v}(\xi) \geq \eta$.

To define the branching measure $\mu$ on $\partial T$ we need a limiting operation. We define

$$
\mu(B(v))=\lim _{k \rightarrow \infty} \frac{Z_{k}(v)}{Z_{k+n}},
$$

whenever $v \in T$ is in generation $n$. Martingale arguments, which are detailed in Section 3.2, show that this limit exists almost surely and $\mu(B(v))$ is nonzero for every vertex $v \in T$. The $\mu$ such defined is a probability measure on $\partial T$ and is called the branching measure or limit uniform measure in the terminology of [23] and [22]. Liu [19] showed in this setting that, almost surely,

$$
\underline{\operatorname{dim}}_{\mu}(\xi)=\mathfrak{a} \text { for all } \xi \in \partial T,
$$

so that only for the upper local dimension we may see a nontrivial spectrum. To ensure this, we assume that the Galton-Watson tree is of Schröder type, i.e. $\mathrm{P}(N=1)>0$. Under this condition, it was shown by Shieh and Taylor [37] and Mörters and Shieh [28] that, with

$$
\tau:=-\frac{\log \mathrm{P}(N=1)}{\mathfrak{a}}
$$

for all $\mathfrak{a} \leq \theta \leq \mathfrak{a}(1+1 / \tau)$, almost surely,

$$
\operatorname{dim}\left\{\xi \in \partial T: \overline{\operatorname{dim}}_{\mu}(\xi)=\theta\right\}=\mathfrak{a}\left(\frac{\mathfrak{a}}{\theta}(1+\tau)-\tau\right) .
$$

Again, we get the same result when we replace the condition $\overline{\operatorname{dim}}_{\mu}(\xi)=\theta$ on the left-hand side by $\overline{\operatorname{dim}}_{\mu}(\xi) \geq \theta$. If $\theta>\mathfrak{a}(1+1 / \tau)$, almost surely, the set on the left-hand side is empty; however, if $\theta=\mathfrak{a}(1+1 / \tau)$, it is almost surely nonempty. 


\subsection{Statement of the main results}

In the light of the previous results it is natural to ask for the Hausdorff dimension of the intersections of the exceptional sets for the visibility and branching measures, i.e. for

$$
\operatorname{dim}\left\{\xi \in \partial T: \operatorname{dim}_{v}(\xi)=\eta, \overline{\operatorname{dim}}_{\mu}(\xi)=\theta\right\}
$$

when $\mathfrak{a}<\theta \leq \mathfrak{a}(1+1 / \tau)$. This set, however, is empty for any value of $\eta$. Indeed, for rays with untypically large upper local dimension of the branching measure, the local dimension of the visibility measure cannot exist. This is stated more precisely in our first theorem.

Theorem 2.1. Let $\theta>\mathfrak{a}$. Then, almost surely, for all $\xi \in \partial T$, we have

$$
\overline{\operatorname{dim}}_{\mu}(\xi) \geq \theta \quad \text { implies that } \overline{\operatorname{dim}}_{v}(\xi) \geq \frac{\theta}{\mathfrak{a}} \underline{\operatorname{dim}}_{v}(\xi),
$$

and, therefore, $\operatorname{dim}_{v}(\xi)$ does not exist.

Therefore, in order to obtain an interesting spectrum, we have to look at the upper and lower local dimensions with respect to the visibility measure separately. As our condition on the upper local dimension of the branching measure involves only unusual behaviour at exceptional scales, in order to enforce a nontrivial interaction of the local behaviour of the two measures, we have to look at conditions on the local behaviour of the visibility measure involving unusual behaviour at all scales. We therefore look at the sets

$$
\bar{G}(\theta, \eta)=\left\{\xi \in \partial T: \overline{\operatorname{dim}}_{\mu}(\xi) \geq \theta, \overline{\operatorname{dim}}_{v}(\xi) \leq \eta\right\}
$$

and

$$
\underline{G}(\theta, \eta)=\left\{\xi \in \partial T: \overline{\operatorname{dim}}_{\mu}(\xi) \geq \theta, \underline{\operatorname{dim}}_{v}(\xi) \geq \eta\right\} .
$$

Our main result gives the Hausdorff dimension of these sets. For its formulation, define

$$
\eta_{\text {typ }}^{-}:=\frac{\mathfrak{a}}{\theta} \eta_{\text {typ }}
$$

Theorem 2.2. For $\mathfrak{a} \leq \theta \leq \mathfrak{a}(1+1 / \tau)$ and $\eta \geq 0$, the following statements hold almost surely.

(a) If $\eta \leq \eta_{\text {typ }}$ then

$$
\operatorname{dim} \bar{G}(\theta, \eta)=-\mathfrak{a} \tau\left(1-\frac{\mathfrak{a}}{\theta}\right)+\frac{\mathfrak{a}}{\theta}\left(\inf _{\beta}\{-\beta \eta-\gamma(\beta)\}\right)
$$

if the right-hand side is nonnegative.

(b) If $\eta \geq \eta_{\mathrm{typ}}^{-}$then

$$
\operatorname{dim} \underline{G}(\theta, \eta)=-\mathfrak{a} \tau\left(1-\frac{\mathfrak{a}}{\theta}\right)+\frac{\mathfrak{a}}{\theta}\left(\inf _{\beta}\left\{-\beta \eta \frac{\theta}{\mathfrak{a}}-\gamma(\beta)\right\}\right)
$$

if the right-hand side is nonnegative.

Remark 2.1. The simultaneous multifractal spectrum produces a swallowtail-shaped spectrum for the visibility measure in the presence of a nontrivial condition on the branching measure. This can be seen in Figure 1 . If we consider values of $\theta>\mathfrak{a}$, it follows that $\eta_{\text {typ }}^{-}<\eta_{\text {typ }}$ and the left and right branches of the spectrum overlap. 


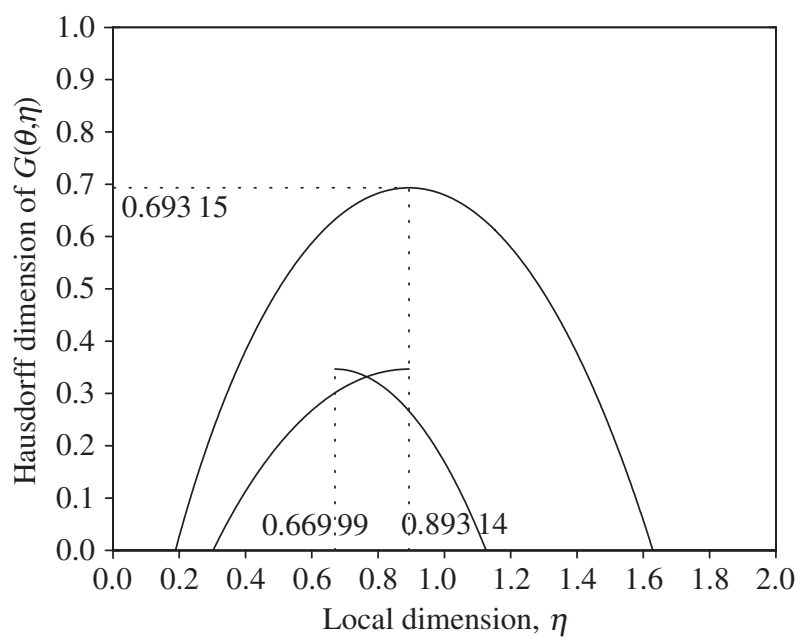

FIGURE 1: Multifractal spectrum of the visibility measure on a Galton-Watson tree with geometric offspring random variable with $p=0.5$. The top curve provides the multifractal spectrum when $\theta=\mathfrak{a}$, while the bottom curves provide the multifractal spectrum when $\theta=\frac{4}{3} \mathfrak{a}$. Here the left branch corresponds to $\operatorname{dim} \bar{G}(\theta, \eta)$, while the right branch corresponds to $\operatorname{dim} \underline{G}(\theta, \eta)$. The two branches overlap at the point

$$
\left(\eta_{\text {typ }}^{-}, \eta_{\text {typ }}\right) \approx(0.67,0.89) \text {. }
$$

Remark 2.2. Considering values of the local dimension of the branching measure equal to the typical local dimension (i.e. taking $\theta=\mathfrak{a}$ ) in Theorem 2.2(a) and (b) leads to a variant of the multifractal spectrum for the upper and lower local dimensions of the visibility measure, respectively. Similarly, setting $\eta=\eta_{\text {typ }}$ in Theorem 2.2(a) and $\eta=\eta_{\text {typ }}^{-}$in Theorem 2.2(b) yields a variant of the spectrum for the branching measure. We conjecture that in the definitions of $\bar{G}(\theta, \eta)$ and $\underline{G}(\theta, \eta)$ all inequality signs can be replaced by equalities without impairing the statement of Theorem 2.2. However, our proof techniques are not strong enough to verify this.

The remainder of the paper is devoted to the proofs of our two main results, given in Sections 3 and 4 . Each section starts with a heuristic explanation of the result and a sketch of the main tools of the proof.

\section{Proof of Theorem 2.1}

\subsection{Heuristics}

If, for some $\lambda>1$, the ray $\xi$ contains a substring $\xi_{n}, \ldots, \xi_{\lambda n}$ consisting of vertices with only one offspring, the balls $B\left(\xi_{n}\right)$ and $B\left(\xi_{\lambda n}\right)$ are equal. Hence, the ratio $\log \mu B(\xi, r) / \log r$ at $r=\mathrm{e}^{-n}$ is, by a factor $\lambda$, larger than at $r=\mathrm{e}^{-n \lambda}$. Therefore, a ray with infinitely many such substrings typically has $\overline{\operatorname{dim}}_{\mu}(\xi) \geq \mathfrak{a} \lambda$.

Conversely, a ray $\xi$ with $\overline{\operatorname{dim}}_{\mu}(\xi) \geq \theta$ typically has infinitely many substrings $\xi_{n}, \ldots, \xi_{(\theta / \mathfrak{a}) n}$ consisting of vertices with predominantly only one offspring. In this case, the visibility measure of the balls $B\left(\xi, \mathrm{e}^{-n}\right)$ and $B\left(\xi, \mathrm{e}^{-n \theta / \mathfrak{a}}\right)$ is nearly equal, so that the ratio $\log \nu B(\xi, r) / \log r$ drops by a factor of $\mathfrak{a} / \theta$ when $r$ moves down from $\mathrm{e}^{-n}$ to $\mathrm{e}^{-n \theta / \mathfrak{a}}$. Therefore, if $\overline{\operatorname{dim}}_{v}(\xi)=\eta$, following the radii $r_{n}=\mathrm{e}^{-n \theta / \mathfrak{a}}$ produces a sequence of radii with $\lim \log \nu B\left(\xi, r_{n}\right) / \log r_{n} \leq \eta \mathfrak{a} / \theta$. 
The proof follows these heuristics closely. Lemma 3.3, below, shows that if a vertex $v$ in the $n$th generation has very little offspring for the next $(\theta / \mathfrak{a}-1) n$ generations, this implies a lower bound for $v B(w)$ for each of these offspring vertices $w$. In Lemma 3.4, below, which will also be crucial in the proof of the upper bound in Theorem 2.2, we show that a ray $\xi$ with $\overline{\operatorname{dim}}_{\mu}(\xi) \geq \theta$ has infinitely many vertices $\xi_{n}$ which have very little offspring for the next $(\theta / \mathfrak{a}-1) n$ generations. Combining these two ingredients completes the proof.

\subsection{Proof of Theorem 2.1}

We start with two very elementary lemmas.

Lemma 3.1. Let $\left\{a_{n}\right\}_{n=1}^{\infty}$ and $\left\{b_{n}\right\}_{n=1}^{\infty}$ be real sequences, and let $\vartheta>0$. Then

$$
\limsup _{n \rightarrow \infty} a_{n}<\vartheta \liminf _{n \rightarrow \infty} b_{n}
$$

implies that, for all $\varepsilon>0$, there exists an $n_{0} \in \mathbb{N}$ such that, for all $n \geq n_{0}$, we have $a_{n}<(\vartheta+\varepsilon) b_{n}$.

Proof. Suppose that there exists a $\varepsilon>0$ and a sequence $n_{k} \uparrow \infty$ such that $a_{n_{k}} \geq(\vartheta+\varepsilon) b_{n_{k}}$. Then

$$
\limsup _{n \rightarrow \infty} a_{n} \geq \limsup _{k \rightarrow \infty} a_{n_{k}} \geq(\vartheta+\varepsilon) \limsup _{k \rightarrow \infty} b_{n_{k}} \geq(\vartheta+\varepsilon) \liminf _{k \rightarrow \infty} b_{n_{k}} \geq(\vartheta+\varepsilon) \liminf _{n \rightarrow \infty} b_{n},
$$

which is a contradiction.

Lemma 3.2. For all $0 \leq j \leq k-1$, suppose that $n_{j} \in \mathbb{N}$. Then, for every $n \in \mathbb{N}$,

$$
\sum_{j=0}^{k-1}\left(n_{j}-1\right) \leq n \quad \text { implies that } \prod_{j=0}^{k-1} n_{j} \leq \mathrm{e}^{n} .
$$

Proof. From the fact that $\log x \leq x-1$ for all $x>0$ we have

$$
n \geq \sum_{j=0}^{k-1}\left(n_{j}-1\right) \geq \sum_{j=0}^{k-1} \log n_{j}=\log \prod_{j=0}^{k-1} n_{j} .
$$

Hence the result follows by taking the exponent of both sides.

The next lemma makes precise the intuition that in a tree with little offspring after $k$ generations, the visibility measure of any vertex in generation $k$ must not be small.

Lemma 3.3. If $k \in \mathbb{N}$ and $Z_{k} \leq n$, then, for all $w \in T$ with $|w|=k$, we have $v(B(w)) \geq \mathrm{e}^{-n}$.

Proof. Suppose that $Z_{k} \leq n$, and let $w=\left(w_{0}, \ldots, w_{k}\right) \in T$ be a vertex in the $k$ th generation. As every vertex has always at least one offspring in any later generation, we observe that

$$
\sum_{j=0}^{k-1}\left(N\left(w_{j}\right)-1\right) \leq Z_{k} \leq n,
$$

and so by Lemma 3.2 we have $\prod_{j=0}^{k-1} N\left(w_{j}\right) \leq \mathrm{e}^{n}$. Hence $v(B(w))=\prod_{j=0}^{k-1} N\left(w_{j}\right)^{-1} \geq \mathrm{e}^{-n}$. 
We now recall the relationship of the branching measure $\mu$ and martingales. Given a vertex $v \in T$, the process $\left(Z_{k}(v) / \mathfrak{m}^{k}: k \in \mathbb{N}\right)$ is a martingale, which, by the Kesten-Stigum theorem, is uniformly integrable if and only if $\mathrm{E} N \log N<\infty$. This condition is satisfied under our moment assumptions and, therefore, there exist random variables $(W(v): v \in T)$ such that

$$
\lim _{k \rightarrow \infty} \frac{Z_{k}(v)}{\mathfrak{m}^{k}}=W(v) \quad \text { almost surely, and } \mathbb{E} W(v)=1
$$

Abbreviating $W:=W(\rho)$ we get, from the definition of the branching measure,

$$
\mu(B(v))=\lim _{k \rightarrow \infty} \frac{Z_{k}(v)}{Z_{k+n}}=\frac{W(v)}{W \mathfrak{m}^{n}} \quad \text { for all } v \in T,|v|=n,
$$

and, moreover, for every $k \in \mathbb{N}$, we obtain the decomposition

$$
W(v)=\frac{1}{\mathfrak{m}^{k}} \sum_{\substack{w \in T \\|w|=k}} W(w),
$$

where the $(W(w):|w|=k)$ are independent with the same distribution as $W$. From this formula and the fact that $W>0$ with positive probability, it is easy to see that $W>0$ almost surely.

Our final lemma makes precise the intuition that a ray $\xi$ with $\overline{\operatorname{dim}}_{\mu}(\xi) \geq \theta$ has infinitely many vertices $\xi_{n}$ with very little offspring for the next $(\theta / \mathfrak{a}-1) n$ generations. A weaker form of this lemma appears in Lemma 5.3 of [37].

Lemma 3.4. Let $\theta \geq \mathfrak{a}$ and $\varepsilon>0$. Then, almost surely, there exist only finitely many vertices $v \in T$ such that

$$
\frac{\log \mu(B(v))}{-|v|} \geq \theta-\varepsilon \text { and } Z_{k}(v)>\varepsilon|v|,
$$

where $k=k(|v|, \varepsilon)$ is defined by

$$
k(n, \varepsilon)=\left\lfloor n\left(\frac{\theta}{\mathfrak{a}}-1-\frac{\varepsilon}{\mathfrak{a}}\right)-A(\varepsilon)\right\rfloor
$$

for a suitable constant $A(\varepsilon)$.

Proof. Since $W>0$ almost surely, we can choose $A(\varepsilon)>0$ such that

$$
\mathbb{P}\left(W<\mathfrak{m}^{-A(\varepsilon)}\right) \leq \mathrm{e}^{-2 \mathfrak{a} / \varepsilon} .
$$

Using (3.1), it suffices to show that

$$
\mathbb{P}\left(\frac{\log W(v)}{-|v|} \geq \theta-\mathfrak{a}-\varepsilon \text { and } Z_{k}(v)>\varepsilon|v|\right)
$$

is summable over the vertices of the tree, in order to use the Borel-Cantelli lemma. As the event that $v \in T$ is itself a random event, we have to embed the tree $T$ into the space $\bigcup_{n=0}^{\infty} \mathbb{N}^{n}$ using the canonical address of every vertex, so that the set of all vertices in the $n$th generation is a subset of $\mathbb{N}^{n}$. It follows that the sum of probabilities in (3.4) is equal to

$$
\sum_{n=1}^{\infty} \sum_{v \in \mathbb{N}^{n}} \mathbb{P}\left(W(v) \leq \mathrm{e}^{-n(\theta-\mathfrak{a}-\varepsilon)}, Z_{k}(v)>\varepsilon n\right) \mathbb{P}(v \in T) .
$$


We can estimate

$$
\begin{aligned}
\mathbb{P}\left(W \leq \mathrm{e}^{-n(\theta-\mathfrak{a}-\varepsilon)}, Z_{k}>\varepsilon n\right) & =\mathbb{P}\left(\mathfrak{m}^{-k} \sum_{\substack{w \in T \\
|w|=k}} W(w) \leq \mathfrak{m}^{-n(\theta / \mathfrak{a}-1-\varepsilon / \mathfrak{a})}, Z_{k}>\varepsilon n\right) \\
& \leq \mathbb{P}\left(\sum_{j=1}^{\lceil\varepsilon\rceil} W_{j} \leq \mathfrak{m}^{-A(\varepsilon)}, Z_{k}>\varepsilon n\right)
\end{aligned}
$$

where $W_{1}, W_{2}, \ldots$ are independent copies of $W$. Using this independence,

$$
\mathbb{P}\left(\sum_{j=1}^{\lceil\varepsilon n\rceil} W_{j} \leq \mathfrak{m}^{-A(\varepsilon)}, Z_{k}>\varepsilon n\right) \leq \mathbb{P}\left(W \leq \mathfrak{m}^{-A(\varepsilon)}\right)^{\lceil\varepsilon n\rceil} \leq \mathrm{e}^{-2 n \mathfrak{a}},
$$

where the last inequality holds by the definition of $A(\varepsilon)$. Hence, we have

$$
\begin{gathered}
\sum_{n=1}^{\infty} \sum_{v \in \mathbb{N}^{n}} \mathbb{P}\left(W(v) \leq \mathrm{e}^{-n(\theta-\mathfrak{a}-\varepsilon)}, Z_{k}(v)>\varepsilon n\right) \mathbb{P}(v \in T) \\
\leq \sum_{n=1}^{\infty} \mathrm{e}^{-2 n \mathfrak{a}} \underbrace{\sum_{v \in \mathbb{N}^{n}} \mathbb{P}(v \in T)}_{=\mathbb{E} Z_{n}}=\sum_{n=1}^{\infty} \mathrm{e}^{-n \mathfrak{a}}<\infty,
\end{gathered}
$$

and the result follows from the Borel-Cantelli lemma.

Proof of Theorem 2.1. It suffices to show that, for a fixed $0<\vartheta<\theta / \mathfrak{a}$ and $0<\delta<1$,

$$
A:=\left\{\xi \in \partial T: \overline{\operatorname{dim}}_{\mu}(\xi) \geq \theta, \overline{\operatorname{dim}}_{v}(\xi)<\vartheta \underline{\operatorname{dim}}_{v}(\xi), \underline{\operatorname{dim}}_{v}(\xi)>\delta\right\}=\varnothing .
$$

Let $1>\varepsilon_{1}=\theta / \mathfrak{a}-\vartheta>0$ and $\varepsilon_{2}<\left(\varepsilon_{1} \mathfrak{a}\right) \wedge \delta^{2} \vartheta / 4(2+\vartheta+1 / \mathfrak{a})$. There exists an $N_{2} \in \mathbb{N}$ such that, for all $n \geq N_{2}$,

$$
\frac{n}{n+k\left(n, \varepsilon_{2}\right)}>\left(\frac{\theta}{\mathfrak{a}}-\frac{\varepsilon_{2}}{\mathfrak{a}}\right)^{-1}-\frac{1}{2} \delta
$$

Now let $\xi \in A$. By Lemma 3.4 we see that $Z_{k}\left(\xi_{n}\right) \leq \varepsilon_{2} n$ infinitely often. Since $\underline{\operatorname{dim}}_{v}(\xi)>\delta$, there exists an $N_{3} \in \mathbb{N}$ such that, for all $n \geq N_{3}$, we have

$$
\frac{\log v\left(B\left(\xi_{n}\right)\right)}{-n}>\frac{1}{2} \delta
$$

Finally, by Lemma 3.1, there exists an $N_{4} \in \mathbb{N}$ such that, for all $n \geq N_{4}$, we have

$$
\frac{\log v\left(B\left(\xi_{n}\right)\right)}{-n}<\left(\vartheta+\varepsilon_{3}\right) \frac{\log v\left(B\left(\xi_{n+k}\right)\right)}{-(n+k)}
$$

for $\varepsilon_{3}=\varepsilon_{1}-\varepsilon_{2} / \mathfrak{a}>0$. Let $N_{1}=\max \left\{N_{2}, N_{3}, N_{4}\right\}$. For $n \geq N_{1}$, we thus have, by (3.5) and (3.7),

$$
v\left(B\left(\xi_{n}\right)\right)>\exp \left\{\left(\left(\vartheta+\varepsilon_{3}\right)^{-1}-\frac{1}{2} \delta\right)\left(\vartheta+\varepsilon_{3}\right) \log v\left(B\left(\xi_{n+k}\right)\right)\right\} .
$$


For $v \in T$, we denote by $v_{v}$ the visibility measure on the tree $T(v)$. Hence

$$
v\left(B\left(\xi_{n}\right)\right)>v\left(B\left(\xi_{n+k}\right)\right)^{1-\delta\left(\vartheta+\varepsilon_{3}\right) / 2}=\left(\nu\left(B\left(\xi_{n}\right)\right) \nu_{\xi_{n}}\left(B\left(\xi_{k}\right)\right)\right)^{1-\delta\left(\vartheta+\varepsilon_{3}\right) / 2} .
$$

It follows that

$$
v\left(B\left(\xi_{n}\right)\right)^{\delta\left(\vartheta+\varepsilon_{3}\right) / 2}>v_{\xi_{n}}\left(B\left(\xi_{k_{n}}\right)\right)^{1-\delta\left(\vartheta+\varepsilon_{3}\right) / 2}
$$

From (3.6) we have

$$
\nu\left(B\left(\xi_{n}\right)\right)^{\delta\left(\vartheta+\varepsilon_{3}\right) / 2} \leq \mathrm{e}^{-n \delta^{2}\left(\vartheta+\varepsilon_{3}\right) / 4},
$$

and from Lemma 3.3 we have, for infinitely many $n$,

$$
\nu_{\xi_{n}}\left(B\left(\xi_{k}\right)\right)^{1-\delta\left(\vartheta+\varepsilon_{3}\right) / 2} \geq \mathrm{e}^{-n\left(\varepsilon_{2}+\varepsilon_{2} \delta\left(\vartheta+\varepsilon_{3}\right) / 2\right)} .
$$

Combining (3.8) with (3.9) and (3.10) we have $\frac{1}{4} \delta^{2}\left(\vartheta+\varepsilon_{3}\right)<\varepsilon_{2}+\frac{1}{2} \varepsilon_{2} \delta\left(\vartheta+\varepsilon_{3}\right)$, and a small calculation shows that this is in contradiction with the conditions on $\varepsilon_{2}$. Hence, $A=\varnothing$ and the proof is complete.

\section{Proof of Theorem 2.2}

\subsection{Heuristics}

Recall that to satisfy $\overline{\operatorname{dim}}_{\mu}(\xi) \geq \theta$ the ray $\xi$ typically has infinitely many substrings $\xi_{n}, \ldots, \xi_{\theta n / \mathfrak{a}}$ in which each vertex has exactly one offspring. The probability that a fixed vertex $v$ is the first one in such a string is

$$
\mathrm{P}(N=1)^{(\theta / \mathfrak{a}-1) n}=\exp \{-\tau(\theta-\mathfrak{a}) n\} .
$$

Looking at generation $n$, for large $n$, by the dimension spectrum for the visibility measure, there are about

$$
\exp \left\{n \inf _{\beta}\{-\eta \beta-\gamma(\beta)\}\right\}
$$

vertices $v$ which satisfy $v\left(B\left(v_{i}\right)\right) \approx \mathrm{e}^{-i \eta}$ for all $1 \leq i \leq n$. By the law of large numbers we therefore expect that in a large generation $n$ we have about

$$
\exp \left\{n\left(-\tau(\theta-\mathfrak{a})+\inf _{\beta}\{-\eta \beta-\gamma(\beta)\}\right)\right\}
$$

vertices $v$ satisfying these two constraints.

Now look at $\bar{G}(\theta, \eta)$ for $\eta \geq \eta_{\text {typ }}$. If a vertex $v$ satisfies the two constraints above then its single descendants $v_{i}$ in generations $i=n+1, \ldots, \theta n / \mathfrak{a}$ all satisfy the required lower bound $v\left(B\left(v_{i}\right)\right) \geq \mathrm{e}^{-i \eta}$. Therefore, in generation $\theta n / \mathfrak{a}$ we expect about

$$
\exp \left\{n\left(-\tau(\theta-\mathfrak{a})+\inf _{\beta}\{-\eta \beta-\gamma(\beta)\}\right)\right\}
$$

vertices satisfying the constraint on the branching measure for the radius $\mathrm{e}^{-n}$ and the constraint on the visibility measure for all radii $1 \geq r \geq \mathrm{e}^{-n \theta / \mathfrak{a}}$. This procedure can now be applied independently to all the subtrees descending from these vertices, so that we expect the Hausdorff dimension of $\bar{G}(\theta, \eta)$ to be

$$
\frac{\mathfrak{a}}{\theta}\left(-\tau(\theta-\mathfrak{a})+\inf _{\beta}\{-\eta \beta-\gamma(\beta)\}\right) .
$$


Next look at $\underline{G}(\theta, \eta)$ for $\eta \leq \eta_{\text {typ }}^{-}$. If a vertex $v$ satisfies the two constraints above then its only descendant $w$ in generation $\theta n / \mathfrak{a}$ has $v(B(w)) \approx \mathrm{e}^{-n \eta}$, which violates the condition on the visibility measure if $\theta>\mathfrak{a}$. We therefore require that the vertex $v$ satisfies $v\left(B\left(v_{i}\right)\right) \approx \mathrm{e}^{-i \tilde{\eta}}$ for $1 \leq i \leq n$ and $\tilde{\eta}=\theta \eta / \mathfrak{a}$, to ensure that all descendants $v_{i}$ in generations $i=n+$ $1, \ldots, \theta n / \mathfrak{a}$ satisfy the required lower bound $v\left(B\left(v_{i}\right)\right) \geq \mathrm{e}^{-i \eta}$. In generation $\theta n / \mathfrak{a}$ there are now $\exp \left\{n\left(-\tau(\theta-\mathfrak{a})+\inf _{\beta}\{-\tilde{\eta} \beta-\gamma(\beta)\}\right)\right\}$ vertices $w$ satisfying the constraint on the branching measure for the radius $\mathrm{e}^{-n}$ and the constraint on the visibility measure for all radii $1 \geq r \geq \mathrm{e}^{-n \theta / \mathfrak{a}}$. Applying the same procedure independently to all the subtrees descending from these vertices, we now expect the Hausdorff dimension of $\underline{G}(\theta, \eta)$ to be

$$
\frac{\mathfrak{a}}{\theta}\left(-\tau(\theta-\mathfrak{a})+\inf _{\beta}\left\{-\frac{\theta}{\mathfrak{a}} \eta \beta-\gamma(\beta)\right\}\right) .
$$

On a more technical level, we need to treat upper and lower bounds for the Hausdorff dimension separately. For the upper bounds, given in Section 4.2, we use natural coverings of the sets $\bar{G}(\theta, \eta)$ and $\underline{G}(\theta, \eta)$. The sets are covered by balls $B\left(\xi_{\theta n / \mathfrak{a}}\right)$, where $\xi_{n}, \ldots, \xi_{\theta n / \mathfrak{a}}$ are strings of vertices with (mostly) one offspring, and

- in the first case we require that $\nu B\left(\xi_{n}\right) \geq \mathrm{e}^{-n \eta}$,

- in the second case we require that $\nu B\left(\xi_{\theta n / \mathfrak{a}}\right) \leq \mathrm{e}^{-n \eta \theta / \mathfrak{a}}$.

For the lower bounds, proved in Section 4.3, we use the percolation technique suggested in [21] and used in [28] to study the branching measure. For a given retention parameter $p \in[0,1]$, we remove any edge from the tree independently with probability $1-p$ and consider the connected component of the root. If this connected component has a positive probability of being infinite, we obtain a lower bound on the Hausdorff dimension of the boundary of the tree, given in terms of $p$. In our case we need to establish a positive probability for the existence of a ray, which survives percolation and satisfies the constraints on the visibility measure for balls of every radius, and the constraints on the branching measure for infinitely many radii. The technical construction of this ray is based on the idea given in the heuristics above and a density argument, which uses the Baire category theorem in a carefully constructed regular subset of $\partial T$.

\subsection{The upper bounds}

Given $\delta>0$ and $\eta \leq \eta_{\text {typ }}$, we cover $\bar{G}(\theta, \eta)$ with balls of radii less than or equal to $\delta$. To do this, take $n_{0}=\lceil-\log \delta\rceil$, let $\varepsilon>0$, and let $k$ be as defined in (3.3). By Lemma 3.4 we have a cover for $\bar{G}(\theta, \eta)$ given by

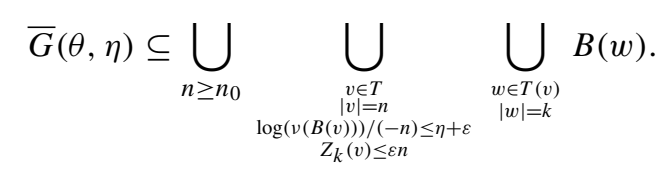

Similarly, if $\eta \geq \eta_{\text {typ }}^{-}$, we have a cover for $\underline{G}(\theta, \eta)$ given by

$$
\underline{G}(\theta, \eta) \subseteq \bigcup_{n \geq n_{0}} \bigcup_{\substack{v \in T \\
|v|=n \\
Z_{k}(v) \leq \varepsilon n}} \bigcup_{\begin{array}{c}
w \in T(v) \\
|w|=k \\
\log v(B(w)) /(-(n+k)) \geq \eta-\varepsilon
\end{array}} B(w) .
$$

Note that, in line with the heuristics, in (4.1) the condition on the visibility measure is tested for the vertices in generation $n$, whereas in (4.2) it is tested for the vertices in generation $n+k$. 
Verification that the covers above give good upper bounds is very similar, and, therefore, we focus on (4.2).

We require the following lemma, which is a combination of two results from the literature.

Lemma 4.1. If $n_{k} / \mathfrak{m}^{k} \rightarrow 0$ as $k \rightarrow \infty$ then $\mathbb{P}\left(Z_{k} \leq n_{k}\right)=\exp \{-k \mathfrak{a} \tau+o(k)\}$.

Proof. By Theorem 4 of [12] we have

$$
\mathbb{P}\left(Z_{k} \leq n_{k}\right)=\mathbb{P}\left(W<\mathfrak{m}^{-k} n_{k}\right)(1+o(1)),
$$

and a well-known result of [10], see also [7] and [27], states that there exist constants $c, C>0$ such that, for $0<\varepsilon<1$, we have

$$
c \varepsilon^{\tau} \leq \mathbb{P}(W<\varepsilon) \leq C \varepsilon^{\tau} .
$$

Hence, $\mathbb{P}\left(Z_{k} \leq n_{k}\right) \leq C(1+o(1))\left(\mathfrak{m}^{-k} n_{k}\right)^{\tau}=\exp \{-k \mathfrak{a} \tau+o(k)\}$, and analogously for the lower bound.

We need to bound the sum of diameters, raised to the power $s$, taken over all the covering balls, i.e.

$$
\sum_{n \geq n_{0}} \sum_{\substack{v \in T \\|v|=n}} 1\left\{Z_{k}(v) \leq \varepsilon n\right\} \sum_{\substack{w \in T(v) \\|w|=k}} 1\left\{v(B(w)) \leq \mathrm{e}^{-(n+k)(\eta-\varepsilon)}\right\}|B(w)|^{s} .
$$

To determine when this quantity is finite, we take its expectation, which equals

$$
\sum_{n \geq n_{0}} \mathrm{e}^{-(n+k) s} \mathbb{E}\left[\sum_{\substack{w \in T \\|w|=n+k}} \mathbf{1}\left\{Z_{k}\left(w_{n}\right) \leq \varepsilon n\right\} \mathbf{1}\left\{v(B(w)) \leq \mathrm{e}^{-(n+k)(\eta-\varepsilon)}\right\}\right] .
$$

Now note that, for any vertex $w \in T$ with $|w|=n+k$, we have $\sum_{j=n}^{n+k-1}\left(N\left(w_{j}\right)-1\right) \leq$ $Z_{k}\left(w_{n}\right)$, and, hence, by Lemma 3.2, $Z_{k}\left(w_{n}\right) \leq \varepsilon n$ implies that

$$
\prod_{j=n}^{n+k-1} N\left(w_{j}\right) \leq \mathrm{e}^{\varepsilon n}
$$

Now, for $\beta>0$, it follows that the expectation of interest is equal to

$$
\begin{gathered}
\sum_{n \geq n_{0}} \mathrm{e}^{-(n+k) s} \mathbb{E}\left[\sum_{\substack{w \in T \\
|w|=n+k}} \mathbf{1}\left\{Z_{k}\left(w_{n}\right) \leq \varepsilon n, \prod_{j=n}^{n+k-1} N\left(w_{j}\right) \leq \mathrm{e}^{\varepsilon n}, v(B(w)) \leq \mathrm{e}^{-(n+k)(\eta-\varepsilon)}\right\}\right] \\
=\sum_{n \geq n_{0}} \mathrm{e}^{-(n+k) s} \mathbb{E}\left[\sum _ { \substack { w \in T \\
| w | = n + k } } \mathbf { 1 } \left\{Z_{k}\left(w_{n}\right) \leq \varepsilon n, \prod_{j=n}^{n+k-1} N\left(w_{j}\right)^{\beta} \leq \mathrm{e}^{\beta \varepsilon n},\right.\right. \\
\leq \sum_{n \geq n_{0}} \mathrm{e}^{-(n+k) s} \mathbb{E}\left[\sum_{\substack{w \in T \\
|w|=n+k}}^{n+k-1} \mathbf{1}\left\{Z_{k}\left(w_{n}\right) \leq \varepsilon n, \prod_{j=0}^{n-1} N\left(w_{j}\right)^{\beta} \geq \mathrm{e}^{\beta(n+k)(\eta-\varepsilon)-\beta \varepsilon n}\right\}\right] .
\end{gathered}
$$


Expressing the sum over the vertices in generation $n+k$ as a sum over the vertices in generation $n$, we see that this is equal to

$$
\begin{aligned}
& \sum_{n \geq n_{0}} \mathrm{e}^{-(n+k) s} \mathbb{E}\left[Z_{k}(v) \sum_{\substack{v \in T \\
|v|=n}} \mathbf{1}\left\{Z_{k}(v) \leq \varepsilon n, \prod_{j=0}^{n-1} N\left(v_{j}\right)^{\beta} \geq \mathrm{e}^{\beta(n+k)(\eta-\varepsilon)-\beta \varepsilon n}\right\}\right] \\
& \quad \leq \sum_{n \geq n_{0}} \mathrm{e}^{-(n+k) s} \mathrm{e}^{\log \varepsilon n} \mathrm{e}^{-\beta(n+k)(\eta-\varepsilon)+\beta \varepsilon n} \mathbb{E}\left[\sum_{\substack{v \in T \\
|v|=n}}^{n-1} \prod_{j=0}^{n} N\left(v_{j}\right)^{\beta} \mathbf{1}\left\{Z_{k}(v) \leq \varepsilon n\right\}\right]
\end{aligned}
$$

The expectation on the right-hand side is equal to, using Lemma 4.1,

$$
\begin{aligned}
\mathbb{E}\left[\sum_{\substack{v \in T \\
|v|=n}} \prod_{j=0}^{n-1} N\left(v_{j}\right)^{\beta} \mathbf{1}\left\{Z_{k}(v) \leq \varepsilon n\right\}\right] & =\mathbb{E}\left[\sum_{\substack{v \in T \\
|v|=n}}^{n-1} N\left(\prod_{j=0}{ }_{j}^{\beta}\right] \mathbb{P}\left\{Z_{k}(v) \leq \varepsilon n\right\}\right. \\
& =\mathrm{e}^{-n \gamma(\beta)-k \mathfrak{a} \tau+o(n)}
\end{aligned}
$$

Summarising, the expectation in (4.3) is finite if

$$
-\beta\left(\frac{\theta}{\mathfrak{a}}-\frac{\varepsilon}{\mathfrak{a}}\right)(\eta-\varepsilon)+\beta \varepsilon-\tau(\theta-\mathfrak{a}-\varepsilon)-\gamma(\beta)<s\left(\frac{\theta}{\mathfrak{a}}-\frac{\varepsilon}{\mathfrak{a}}\right) .
$$

Solving for $s$ and noting that $\varepsilon>0$ was arbitrary, we get

$$
\operatorname{dim} \underline{G}(\theta, \eta) \leq-\mathfrak{a} \tau\left(1-\frac{\mathfrak{a}}{\theta}\right)+\frac{\mathfrak{a}}{\theta} \inf _{\beta>0}\left\{-\frac{\beta \theta \eta}{\mathfrak{a}}-\gamma(\beta)\right\}
$$

It remains to show that the infimum can be extended to nonpositive values of $\beta$.

Lemma 4.2. If $\eta>\eta_{\mathrm{typ}}^{-}$then

$$
\inf _{\beta>0}\left\{-\frac{\beta \theta \eta}{\mathfrak{a}}-\gamma(\beta)\right\}=\inf _{\beta}\left\{-\frac{\beta \theta \eta}{\mathfrak{a}}-\gamma(\beta)\right\} .
$$

Proof. Differentiating the variational problem with respect to $\beta$ we see that

$$
\frac{\mathrm{d}}{\mathrm{d} \beta}\left\{-\frac{\beta \theta \eta}{\mathfrak{a}}-\gamma(\beta)\right\}=-\frac{\theta \eta}{\mathfrak{a}}+\frac{\mathrm{d}}{\mathrm{d} \beta} \log \mathbb{E} N^{\beta+1}=-\frac{\theta \eta}{\mathfrak{a}}+\frac{\mathbb{E} N^{\beta+1} \log N}{\mathbb{E} N^{\beta+1}},
$$

and so the minimizer $\beta_{0}$ satisfies

$$
\frac{\mathfrak{a}}{\theta} \frac{\mathbb{E} N^{\beta_{0}+1} \log N}{\mathbb{E} N^{\beta_{0}+1}}=\eta
$$

The assumption $\eta>\eta_{\text {typ }}^{-}$implies that $\beta_{0}>0$.

Finally, when $\eta=\eta_{\text {typ }}^{-}$, then the minimiser in $\inf _{\beta}\{-\beta \theta \eta / \mathfrak{a}-\gamma(\beta)\}$ is $\beta=0$. Combining this with Lemma 4.2 and (4.4) completes the proof of the upper bound. 


\subsection{The lower bounds}

For the proof of the lower bound, we will again focus on the proof of part (b), as the proof of part (a) is similar but conceptually slightly easier. We use percolation on the tree in order to find a lower bound for the dimension, and first provide a reminder of the notation and important facts.

Given an arbitrary, infinite tree $T$ and a retention parameter $p \in[0,1]$, we define a family of independent $\{0,1\}$-valued random variables $X(v)$, indexed by the vertices, such that $\mathbb{P}(X(v)=$ $1)=p$. The percolated tree $T^{*}$ is then comprised of the connected component of the root in the graph consisting of all the vertices $v \in T$ with $X(v)=1$ and the edges between them. We use the following lemma.

Lemma 4.3. ([21].) Let $T$ be an arbitrary, infinite tree. Then the following statements hold.

(a) If $p<\mathrm{e}^{-\operatorname{dim} \partial T}$ then $\partial T^{*}=\varnothing$ almost surely.

(b) If $p>\mathrm{e}^{-\operatorname{dim} \partial T}$ then $\partial T^{*} \neq \varnothing$ with positive probability.

Remark 4.1. With the help of the Choquet capacitability theorem, we can infer the following useful consequence of Lemma 4.3. If an analytic set $E \subset \partial T$ satisfies $E \cap \partial T^{*} \neq \varnothing$ with positive probability, then $\operatorname{dim} E \geq-\log p$. This is the form in which we shall use Lemma 4.3.

For the visibility measure $v$ on a tree $T$, we define, for any $\eta>0$,

$$
F(\eta):=\left\{\xi \in \partial T: \underline{\operatorname{dim}}_{v}(\xi) \geq \eta\right\}
$$

and a compact set $A(\eta) \subset F(\eta)$ by

$$
A(\eta):=\left\{\xi \in \partial T: v\left(B\left(\xi_{i}\right)\right) \leq \mathrm{e}^{-i \eta} \text { for all } i \in \mathbb{N}\right\} .
$$

The next result is due to Hawkes [15] in a general setup, and proved in the context of trees in the final paragraph of [21].

Lemma 4.4. For any $E \subset \partial T$ and $\varepsilon>0$, we have

$$
\operatorname{dim}\left(E \cap \partial T^{*}\right) \geq \log p+\operatorname{dim} E-\varepsilon \quad \text { with positive probability. }
$$

We now focus our attention on Galton-Watson trees. Recall the Galton-Watson 0-1 law as described in [34]. A property $A$ of trees is called inherited if all finite trees have property $A$ and, whenever $T$ has property $A$, all its descendent subtrees $T(v)$ for $v \in T$ have property $A$. For a Galton-Watson tree, any inherited property $A$ satisfies $\mathbb{P}(A \mid \partial T \neq \varnothing) \in\{0,1\}$.

The next lemma combines the multifractal spectrum for the visibility measure of GaltonWatson trees, as given in [16], with Lemma 4.4. From now on, $\mathbb{P}$ and the notion of probability refer to the joint law of the Galton-Watson tree and the percolation variables indexed by this tree. Observe that, if $T$ is a Galton-Watson tree then so is any percolated tree $T^{*}$ derived from it.

Lemma 4.5. Let

$$
\sup _{\beta<0}\left\{\frac{-\gamma(\beta)}{\beta}\right\}<\eta<\inf _{\beta>0}\left\{\frac{-\gamma(\beta)}{\beta}\right\} .
$$

For every $p \geq \exp \left(-\inf _{\beta}\{-\beta \eta-\gamma(\beta)\}\right)$ and $\varepsilon>0$, we have

$$
\operatorname{dim} A(\eta) \cap \partial T^{*}>\log p+\inf _{\beta}\{-\beta \eta-\gamma(\beta)\}-\varepsilon \quad \text { with positive probability. }
$$


Proof. Recall from [16] that $\operatorname{dim} F(\eta)=\inf _{\beta}\{-\beta \eta-\gamma(\beta)\}$ almost surely. This easily implies that $\operatorname{dim} A(\eta)>\inf _{\beta}\{-\beta \eta-\gamma(\beta)\}-\varepsilon$ with positive probability, and the result follows from Lemma 4.4 .

We now formulate the key lemma for the proof. We define, for $0<x<1$,

$$
A^{x}(\eta):=\left\{\xi \in \partial T: \mathrm{e}^{(\log x / \mathfrak{a}) \eta} v\left(B\left(\xi_{i}\right)\right) \leq \mathrm{e}^{-i \eta} \text { for all } i \in \mathbb{N}\right\} .
$$

Lemma 4.6. Fix $\theta \geq \mathfrak{a}$,

$$
\inf _{\beta>0}\left\{\frac{-\gamma(\beta)}{\beta}\right\}>\tilde{\eta} \geq \eta \geq \eta_{\text {typ }}^{-}
$$

and a retention parameter

$$
p>\exp \left\{\mathfrak{a} \tau\left(1-\frac{\mathfrak{a}}{\theta}\right)-\frac{\mathfrak{a}}{\theta} \inf _{\beta}\{-\beta \tilde{\eta}-\gamma(\beta)\}\right\} .
$$

Then there exist $C, \kappa, \varepsilon_{0}>0$ such that, for all $0<x<1$ and $0<\varepsilon<\varepsilon_{0}$, we have

$$
\begin{aligned}
\mathbb{P}(W & \left.<\kappa x, \operatorname{dim}\left(A^{x}(\eta) \cap F(\tilde{\eta}+\varepsilon) \cap \partial T^{*}\right) \geq\left(1-\frac{\mathfrak{a}}{\theta}\right)\left(\inf _{\beta}\{-\beta \tilde{\eta}-\gamma(\beta)\}+a \tau\right)+2 \varepsilon\right) \\
& \geq C x^{\tau-\log p / \mathfrak{a}} .
\end{aligned}
$$

Proof. Let

$$
k:=k(x):=\left\lceil-\frac{\log x}{\mathfrak{a}}\right\rceil \text { and } \quad d:=\left(1-\frac{\mathfrak{a}}{\theta}\right)\left(\inf _{\beta}\{-\beta \tilde{\eta}-\gamma(\beta)\}+a \tau\right) .
$$

We have, for any $\kappa \geq 1$ and $\varepsilon>0$,

$$
\begin{aligned}
& \mathbb{P}\left(W<\kappa x \text { and } \operatorname{dim}\left(A^{x}(\eta) \cap F(\tilde{\eta}+\varepsilon) \cap \partial T^{*}\right) \geq d+2 \varepsilon\right) \\
& \quad \geq \mathbb{P}\left(W<\kappa x \text { and } \operatorname{dim}\left(A^{x}(\eta) \cap F(\tilde{\eta}+\varepsilon) \cap \partial T^{*}\right) \geq d+2 \varepsilon \mid Z_{k}=1\right) \mathbb{P}\left(Z_{k}=1\right) .
\end{aligned}
$$

Recall that $\mathbb{P}\left(Z_{k}=1\right)=\mathrm{P}(N=1)^{k}$. For a lower bound on the first probability in (4.5), use (3.2) to observe that

$$
\begin{aligned}
& \mathbb{P}\left(W<\kappa x \text { and } \operatorname{dim}\left(A^{x}(\eta) \cap F(\tilde{\eta}+\varepsilon) \cap \partial T^{*}\right) \geq d+2 \varepsilon \mid Z_{k}=1\right) \\
& \quad \geq \mathbb{P}\left(\mathfrak{m}^{-k} \sum_{\substack{w \in T \\
|w|=k}} W(w)<\mathfrak{m}^{-k} \kappa \text { and } \operatorname{dim}\left(A^{x}(\eta) \cap F(\tilde{\eta}+\varepsilon) \cap \partial T^{*}\right) \geq d+2 \varepsilon \mid Z_{k}=1\right) \\
& \quad=\mathbb{P}\left(W(w)<\kappa \text { and } \operatorname{dim}\left(A^{x}(\eta) \cap F(\tilde{\eta}+\varepsilon) \cap \partial T^{*}\right) \geq d+2 \varepsilon \mid Z_{k}=1\right),
\end{aligned}
$$

where $w$ is the unique vertex in the $k$ th generation. Note that $k \eta \geq-(\log x / \mathfrak{a}) \eta$ and, hence, for $0 \leq i \leq k$,

$$
\mathrm{e}^{(\log x / \mathfrak{a}) \eta} v\left(B\left(w_{i}\right)\right) \leq \mathrm{e}^{-k \eta} v\left(B\left(w_{i}\right)\right)=\mathrm{e}^{-k \eta} \leq \mathrm{e}^{-i \eta} .
$$

As $Z_{k}=1$, we have $\partial T^{*} \neq \varnothing$ only if $X\left(w_{i}\right)=1$ for $i=0, \ldots, k$, an event which has probability $p^{k}$. Hence, we see that

$$
\begin{gathered}
\mathbb{P}\left(W(w)<\kappa \text { and } \operatorname{dim}\left(A^{x}(\eta) \cap F(\tilde{\eta}+\varepsilon) \cap \partial T^{*}\right) \geq d+2 \varepsilon \mid Z_{k}=1\right) \\
\geq \mathbb{P}\left(W<\kappa \text { and } \operatorname{dim}\left(A(\eta) \cap F(\tilde{\eta}+\varepsilon) \cap \partial T^{*}\right) \geq d+2 \varepsilon\right) p^{k}
\end{gathered}
$$


As in Lemma 4.5, by the choice of $p$, there exist $c_{0}, \varepsilon_{0}>0$ such that

$$
\mathbb{P}\left(\operatorname{dim}\left(A(\eta) \cap F\left(\tilde{\eta}+\varepsilon_{0}\right) \cap \partial T^{*}\right) \geq d+2 \varepsilon_{0}\right) \geq c_{0} .
$$

Since $W$ is finite, we have

$$
\begin{aligned}
& \mathbb{P}\left(\operatorname{dim}\left(A(\eta) \cap F\left(\tilde{\eta}+\varepsilon_{0}\right) \cap \partial T^{*}\right) \geq d+2 \varepsilon_{0}\right) \\
& \quad=\lim _{\kappa \rightarrow \infty} \mathbb{P}\left(W<\kappa \text { and } \operatorname{dim}\left(A(\eta) \cap F\left(\tilde{\eta}+\varepsilon_{0}\right) \cap \partial T^{*}\right) \geq d+2 \varepsilon_{0}\right) .
\end{aligned}
$$

It follows that, for a large enough choice of $\kappa>1$, we can bound this probability from below by a positive constant $c_{1}>0$. Putting this together with (4.6) we have, for all $0<\varepsilon \leq \varepsilon_{0}$,

$$
\begin{aligned}
\mathbb{P}\left(W<\kappa x \text { and } \operatorname{dim}\left(A^{x}(\eta) \cap F(\tilde{\eta}+\varepsilon) \cap \partial T^{*}\right) \geq d+2 \varepsilon\right) & \geq c_{1} p^{k} \mathrm{P}(N=1)^{k} \\
& \geq C x^{\tau-\log p / \mathfrak{a}}
\end{aligned}
$$

for a suitable constant $C>0$.

We now proceed with the proof of the lower bound. We fix $\theta \geq \mathfrak{a}, \eta \geq \eta_{\mathrm{typ}}^{-}=(\mathfrak{a} / \theta) \eta_{\mathrm{typ}}$, and let $\tilde{\eta}:=\theta \eta / \mathfrak{a}$. We also fix a retention parameter

$$
p>p_{\text {crit }}:=\exp \left\{\mathfrak{a} \tau\left(1-\frac{\mathfrak{a}}{\theta}\right)-\frac{\mathfrak{a}}{\theta} \inf _{\beta}\left\{-\frac{\theta}{\mathfrak{a}} \beta \eta-\gamma(\beta)\right\}\right\},
$$

and denote the percolated tree by $T^{*}$. We define a compact set

$$
A^{*}(\eta):=A(\eta) \cap \partial T^{*}=\left\{\xi \in \partial T^{*}: v\left(B\left(\xi_{n}\right)\right) \leq \mathrm{e}^{-n \eta} \text { for all } n \in \mathbb{N}\right\}
$$

and, for $0<\varepsilon<\varepsilon_{0}$ as in Lemma 4.6, its regularization

$$
\begin{aligned}
A^{\circ}(\eta):=A^{*}(\eta) \backslash \cup\{B(v): \operatorname{dim}(B(v) & \left.\cap A^{*}(\eta) \cap F(\tilde{\eta}+\varepsilon)\right) \\
& \left.<\left(1-\frac{\mathfrak{a}}{\theta}\right)\left(\inf _{\beta}\left\{-\frac{\theta}{\mathfrak{a}} \beta \eta-\gamma(\beta)\right\}+\mathfrak{a} \tau\right)+\varepsilon\right\} .
\end{aligned}
$$

Lemma 4.7. For a suitably small $\varepsilon>0$, we have $A^{\circ}(\eta) \neq \varnothing$ with positive probability.

Proof. It obviously suffices to show that there exists $\varepsilon>0$ such that, with positive probability,

$$
\operatorname{dim}\left(A^{*}(\eta) \cap F(\tilde{\eta}+\varepsilon)\right) \geq\left(1-\frac{\mathfrak{a}}{\theta}\right)\left(\inf _{\beta}\left\{-\frac{\theta}{\mathfrak{a}} \beta \eta-\gamma(\beta)\right\}+\mathfrak{a} \tau\right)+2 \varepsilon .
$$

By Lemma 4.5 we have, with positive probability,

$$
\operatorname{dim}\left(A^{*}(\tilde{\eta}+\varepsilon)\right) \geq \log p+\inf _{\beta}\{-\beta(\tilde{\eta}+\varepsilon)-\gamma(\beta)\}-\varepsilon .
$$

As $p>p_{\text {crit }}$, we find a $\varepsilon>0$ such that

$$
\begin{aligned}
\log p+\inf _{\beta}\{-\beta(\tilde{\eta}+\varepsilon)-\gamma(\beta)\}-\varepsilon & \geq \log p_{\text {crit }}+\inf _{\beta}\{-\beta \tilde{\eta}-\gamma(\beta)\}+2 \varepsilon \\
& =\left(1-\frac{\mathfrak{a}}{\theta}\right)\left(\inf _{\beta}\left\{-\frac{\theta}{\mathfrak{a}} \beta \eta-\gamma(\beta)\right\}+\mathfrak{a} \tau\right)+2 \varepsilon .
\end{aligned}
$$

It remains to note that $\tilde{\eta} \geq \eta$ and, hence, $A^{*}(\eta) \cap F(\tilde{\eta}+\varepsilon) \supseteq A^{*}(\tilde{\eta}+\varepsilon)$. 
We may now consider $0<\varepsilon<\varepsilon_{0}$ fixed to satisfy Lemma 4.7. For each $N \in \mathbb{N}$, let

$$
U(N):=\left\{\xi \in A^{\circ}(\eta): \text { there exists } n \geq N \text { with }-\log \mu\left(B\left(\xi_{n}\right)\right)>n \theta+\log \frac{W}{\kappa}\right\} .
$$

The following lemma is key to proving the lower bound for $\underline{G}(\theta, \eta)$.

Lemma 4.8. Almost surely, for all $N \in \mathbb{N}, U(N)$ is dense in $A^{\circ}(\eta)$.

Proof. We need to show that, for any $v \in T$ with $B(v) \cap A^{\circ}(\eta) \neq \varnothing$, we find a ray $\xi \in B(v) \cap A^{\circ}(\eta)$ and $n \geq N$ with $-\log \mu\left(B\left(\xi_{n}\right)\right)>n \theta+\log (W / \kappa)$. For this purpose, fix $v \in T$ and abbreviate $m=|v|$. Recall that $T(v)$ is the tree consisting of the offspring of $v$ and use the notation $|w|_{v}$ to indicate the generation of $w$ in $T(v)$. Let $g(n)$ be the $\sigma$-algebra generated by the event $\left\{v \in T^{*}\right\}$, the random variable $v(B(v))$, and the tree $T(v)$ up to generation $n$ including the random variables $\left\{X(w): w \in T(v),|w|_{v} \leq n\right\}$.

Let $\mathcal{K}_{n}$ be the collection of vertices $w=\left(w_{0}, \ldots, w_{n+m}\right)$ with $w_{m}=v$ such that

$$
v(B(w)) \leq \mathrm{e}^{-(m+n) \tilde{\eta}} \quad \text { and } \quad v\left(B\left(w_{i}\right)\right) \leq \mathrm{e}^{-i \eta} \quad \text { for all } i \in\{m, \ldots, m+n\} .
$$

Let $K_{n}$ be the cardinality of $\mathcal{K}_{n}$, which is a $\mathcal{G}(n)$-measurable random variable. We define a sequence of $g(n)$ stopping times $N_{0}, N_{1}, \ldots$ by $N_{0}=N$ and

$$
N_{k}=\min \left\{n>N_{k-1}: K_{n} \geq \exp \left\{n\left(\left(1-\frac{\mathfrak{a}}{\theta}\right)\left(\inf _{\beta}\left\{-\frac{\theta}{\mathfrak{a}} \beta \eta-\gamma(\beta)\right\}+\mathfrak{a} \tau\right)+\frac{\varepsilon}{2}\right)\right\}\right\} .
$$

If $v \in T$ satisfies $B(v) \cap A^{\circ}(\eta) \neq \varnothing$ then these stopping times are finite, because the contrary would imply an upper bound of $(1-\mathfrak{a} / \theta)\left(\inf _{\beta}\{-\theta \beta \eta / \mathfrak{a}-\gamma(\beta)\}+\mathfrak{a} \tau\right)+\varepsilon / 2$ for the Hausdorff dimension of $B(v) \cap A^{*}(\eta) \cap F(\tilde{\eta}+\varepsilon)$, contradicting the assumption that $B(v) \cap A^{\circ}(\eta) \neq \varnothing$.

For every vertex $w \in \mathcal{K}_{N_{j}}$, define the sets $A_{w}^{x}(\eta)$ and $F_{w}(\eta)$ in the same way as $A^{x}(\eta)$ and $F(\eta)$, but with reference to the tree $T(w)$. Abbreviate

$$
d:=\left(1-\frac{\mathfrak{a}}{\theta}\right)\left(\inf _{\beta}\left\{-\frac{\theta}{\mathfrak{a}} \beta \eta-\gamma(\beta)\right\}+\mathfrak{a} \tau\right)+2 \varepsilon \quad \text { and } \quad x:=\mathrm{e}^{\left(N_{j}+m\right)(\mathfrak{a}-\theta)} .
$$

Define the event

$$
\begin{aligned}
E(w):= & \left\{-\log \mu(B(w))>\left(N_{j}+m\right) \theta+\log \frac{W}{\kappa}\right. \\
& \text { and } \left.\operatorname{dim}\left(A_{w}^{x}(\eta) \cap F_{w}(\tilde{\eta}+\varepsilon) \cap \partial T(w)^{*}\right) \geq d\right\} \\
= & \left\{W(w)<\kappa x \text { and } \operatorname{dim}\left(A_{w}^{x}(\eta) \cap F_{w}(\tilde{\eta}+\varepsilon) \cap \partial T(w)^{*}\right) \geq d\right\},
\end{aligned}
$$

where the second equality follows from (3.1). Given $g\left(N_{j}\right)$, the events $E(w)$ for $w \in \mathcal{K}_{N_{j}}$ are independent. Moreover, $E(w)$ implies that there exists a ray

$$
\left(w_{1}, \ldots, w_{m+N_{j}}, \xi_{1}, \xi_{2}, \ldots\right) \in B(v) \cap A^{\circ}(\eta)
$$

such that $-\log \mu(B(w))>\left(N_{j}+m\right) \theta+\log (W / \kappa)$, which are the required properties.

It remains to show that, given a vertex $v \in T,|v|=m$, such that $N_{0}, N_{1}, \ldots$ is a sequence of finite stopping times, almost surely, there exist $j \geq 1$ and $w \in \mathcal{K}_{N_{j}}$ such that $E(w)$ holds. 
By Lemma 4.6, the probability $\mathbb{P}\left(E(w) \mid \mathcal{G}\left(N_{j}\right)\right)$ given $w \in \mathcal{K}_{N_{j}}$ is bounded from below by a constant multiple of

$$
\begin{aligned}
& \exp \left\{\left(N_{j}+m\right)(\mathfrak{a}-\theta)\left(\tau-\frac{\log p}{\mathfrak{a}}\right)\right\} \\
& \geq \exp \left\{-\left(N_{j}+m\right)\left(1-\frac{\mathfrak{a}}{\theta}\right)\left(\inf _{\beta}\left\{-\beta \eta \frac{\theta}{\mathfrak{a}}-\gamma(\beta)\right\}+\mathfrak{a} \tau\right)\right\} \\
& \quad=: h\left(N_{j}\right) .
\end{aligned}
$$

Recall that

$$
K_{N_{j}} \geq \exp \left(N_{j}\left(\left(1-\frac{\mathfrak{a}}{\theta}\right)\left(\inf _{\beta}\left\{-\beta \eta \frac{\theta}{\mathfrak{a}}-\gamma(\beta)\right\}+\mathfrak{a} \tau\right)+\frac{\varepsilon}{2}\right)\right)
$$

Hence,

$$
\mathbb{P}\left(E(w) \text { fails for all } w \in \mathcal{K}_{N_{j}} \mid \mathcal{g}\left(N_{j}\right)\right) \leq\left(1-C \exp \left\{h\left(N_{j}\right)\right\}\right)^{K_{N_{j}}},
$$

and the result follows as $\lim _{j \rightarrow \infty}\left(1-C \exp \left\{h\left(N_{j}\right)\right\}\right)^{K_{N_{j}}}=0$.

We now use Lemma 4.8 and the Baire (category) theorem to prove the following lemma, which will eventually lead to a lower bound for $\underline{G}(\theta, \eta)$. Define

$$
\begin{aligned}
A^{*}(\theta, \eta):=\left\{\xi \in \partial T^{*}: \nu\left(B\left(\xi_{n}\right)\right)\right. & \leq \mathrm{e}^{-n \eta} \text { for all } n \in \mathbb{N} \\
& \text { and } \left.\mu\left(B\left(\xi_{n}\right)\right) \leq \frac{\kappa}{W} \mathrm{e}^{-n \theta} \text { for infinitely many } n \in \mathbb{N}\right\} .
\end{aligned}
$$

Lemma 4.9. With positive probability, we have $A^{*}(\theta, \eta) \neq \varnothing$.

Proof. Note that $\bigcap_{N=1}^{\infty} U(N) \subseteq A^{*}(\theta, \eta)$. Observe that $U(N)$ is relatively open in $A^{\circ}(\eta)$, which is compact, and, hence, a complete metric space. Now Lemma 4.8 says that, almost surely, for every $N \in \mathbb{N}, U(N)$ is dense in $A^{\circ}(\eta)$. Therefore, by the Baire theorem,

$$
\bigcap_{N=1}^{\infty} U(N) \text { is dense in } A^{\circ}(\eta)
$$

and, hence, $A^{*}(\theta, \eta)$ is nonempty if $A^{\circ}(\eta) \neq \varnothing$, which, by Lemma 4.7, is an event of positive probability.

We now complete the proof of Theorem 2.2(b). By Lemma 4.9, if $p>p_{\text {crit }}$, with positive probability,

$$
\underline{G}(\theta, \eta) \cap \partial T^{*} \supseteq A^{*}(\theta, \eta) \neq \varnothing .
$$

Hence, we obtain from the remark following Lemma 4.3 that $\operatorname{dim} G(\theta, \eta) \geq-\log p$ with positive probability. Finally, we observe that $\operatorname{dim} \underline{G}(\theta, \eta)<-\log p$ is an inherited property of the Galton-Watson tree and as a result either has probability 0 or 1 by the Galton-Watson $0-1$ law. Since the complementary event has positive probability, it must have probability 0 . Letting $p \downarrow p_{\text {crit }}$ completes the proof. 


\section{Acknowledgements}

This paper contains material from the first author's $\mathrm{PhD}$ thesis. The second author is supported by an Advanced Research Fellowship from EPSRC. We would like to thank Lars Olsen for directing our attention to some relevant papers in the recent analysis literature.

\section{References}

[1] Anh, V. V., Leonenko, N. N. And Shieh, N.-R. (2008). Multifractality of products of geometric OrnsteinUhlenbeck-type processes. Adv. Appl. Prob. 40, 1129-1156.

[2] Arbeiter, M. and PatzschKe, N. (1996). Random self-similar multifractals. Math. Nachr. 181, 5-42.

[3] Barreira, L. ANd Saussol, B. (2001). Variational principles and mixed multifractal spectra. Trans. Amer. Math. Soc. 353, 3919-3944.

[4] Barreira, L., Saussol, B. and Schmeling, J. (2002). Higher-dimensional multifractal analysis. J. Math. Pures Appl. 81, 67-91.

[5] Benzi, R., Paladin, G., Parisi, G. and Vulpiani, A. (1984). On the multifractal nature of fully developed turbulence and chaotic systems. J. Phys. A 17, 3521-3531.

[6] Berestycki, J. (2003). Multifractal spectra of fragmentation processes. J. Statist. Phys. 113, 411-430.

[7] Biggins, J. D. And Bingham, N. H. (1993). Large deviations in the supercritical branching process. Adv. Appl. Prob. 25, 757-772.

[8] Brown, G., Michon, G. And Peyriere, J. (1992). On the multifractal analysis of measures. J. Statist. Phys. 66, 775-790.

[9] Cawley, R. And Mauldin, R. D. (1992). Multifractal decompositions of Moran fractals. Adv. Math. 92, 196236.

[10] Dubuc, M. S. (1971). La densité de la loi-limite d'un processus en cascade expansif. Z. Wahrscheinlichkeitsth. 19, 281-290.

[11] Falconer, K. (2003). Fractal Geometry, 2nd edn. John Wiley, Hoboken, NJ.

[12] Fleischmann, K. and Wachtel, V. (2007). Lower deviation probabilities for supercritical Galton-Watson processes. Ann. Inst. H. Poincaré Prob. Statist. 43, 233-255.

[13] Frisch, U. and Parisi, G. (1985). On the singularity structure of fully developed turbulence. In Proc. Int. Sum. School Enrico Fermi, North Holland, Amsterdam, pp. 84-88.

[14] Halsey, T. C. et al. (1986). Fractal measures and their singularities: the characterization of strange sets. Phys. Rev. A 33, 1141-1151.

[15] Hawkes, J. (1981). Trees generated by a simple branching process. J. London Math. Soc. 24, 373-384.

[16] Kinnison, A. L. (2008). The multifractal spectrum of harmonic measure for forward moving random walks on a Galton-Watson tree. Statist. Prob. Lett. 78, 3114-3121.

[17] Klenke, A. And Mörters, P. (2005). The multifractal spectrum of Brownian intersection local times. Ann. Prob. 33, 1255-1301.

[18] LaU, K.-S. AND NGaI, S.-M. (1999). Multifractal measures and a weak separation condition. Adv. Math. 141, 45-96.

[19] LiU, Q. (2001). Local dimensions of the branching measure on a Galton-Watson tree. Ann. Inst. H. Poincaré Prob. Statist. 37, 195-222.

[20] Liu, Q. AND Rouault, A. (1997). On two measures defined on the boundary of a branching tree. In Classical and Modern Branching Processes (Minneapolis, MN, 1994; IMA Vol. Math. Appl. 84), Springer, New York, pp. 187-201.

[21] LyOns, R. (1990). Random walks and percolation on trees. Ann. Prob. 18, 931-958.

[22] Lyons, R. And Peres, Y. (2010). Probability on Trees and Networks. In preparation. Available at http://php. indiana.edu/ rdlyons/prbtree/prbtree.html.

[23] Lyons, R., Pemantle, R. And Peres, Y. (1995). Ergodic theory on Galton-Watson trees: speed of random walk and dimension of harmonic measure. Ergodic Theory Dynam. Syst. 15, 593-619.

[24] Mandelbrot, B. B. (1974). Intermittent turbulence in self-similar cascades: divergence of high moments and dimension of the carrier. J. Fluid Mech. 62, 331-358.

[25] Mannersalo, P., Norros, I. And Riedi, R. H. (2002). Multifractal products of stochastic processes: construction and some basic properties. Adv. Appl. Prob. 34, 888-903.

[26] Mörters, P. (2009). Why study multifractal spectra? In Trends in Stochastic Analysis (London Math. Soc. Lecture Notes Ser. 353), Cambridge University Press, pp. 99-120.

[27] Mörters, P. And Ortgiese, M. (2008). Small value probabilities via the branching tree heuristic. Bernoulli 14, 277-299.

[28] Mörters, P. ANd Shieh, N.-R. (2004). On the multifractal spectrum of the branching measure of a GaltonWatson tree. J. Appl. Prob. 41, 1223-1229. 
[29] Olsen, L. (1995). A multifractal formalism. Adv. Math. 116, 82-196.

[30] Olsen, L. (2003). Mixed divergence points of self-similar measures. Indiana Univ. Math. J. 52, $1343-1372$.

[31] OLSEN, L. (2003). Multifractal analysis of divergence points of deformed measure theoretical Birkhoff averages. J. Math. Pures Appl. 82, 1591-1649.

[32] Olsen, L. (2005). Mixed generalized dimensions of self-similar measures. J. Math. Anal. Appl. 306, 516-539.

[33] Olsen, L. AND Winter, S. (2003). Normal and non-normal points of self-similar sets and divergence points of self-similar measures. J. London Math. Soc. 67, 103-122.

[34] Peres, Y. (1999). Probability on trees: an introductory climb. In Lectures on Probability Theory and Statistics (Saint-Flour, 1997; Lecture Notes Math. 1717), Springer, Berlin, pp. 193-280.

[35] Perkins, E. A. And Taylor, S. J. (1998). The multifractal structure of super-Brownian motion. Ann. Inst. H. Poincaré Prob. Statist. 34, 97-138.

[36] RAND, D. A. (1989). The singularity spectrum $f(a)$ for cookie-cutters. Ergodic Theory Dynam. Systems 9, 527-541.

[37] SHIEH, N.-R. AND TAYLOR, S. J. (2002). Multifractal spectra of branching measure on a Galton-Watson tree. J. Appl. Prob. 39, 100-111. 